\title{
Ancestor Advantages Prime Better Intellectual Performances_-Evolutionary Psychology-Based Intelligence Priming Effect ${ }^{*}$
}

\author{
Zhang Shu, Guo Qingke \\ Shandong Normal University, Ji'nan, China
}

\begin{abstract}
Research has verified that human beings' intellectual performances can be influenced by intelligence priming. Evolutionary psychology holds that the evolutionary psychological mechanisms which imitate the others, such as intelligence priming, is of survival values. By priming, human beings can not only predict others' complex behaviors, but also accelerate and improve the behaviors that they imitated, that is, to acquire better intellectual performance by use of intelligence priming. This research explores intelligence priming effect from the perspective of evolutionary psychology with both the two experiments employing general intelligence tasks as the inspection method of intelligence priming effect so as to investigate the impacts of ancestors as priming material for the ingroup/outgroup differentiation on the intelligence priming effect. The results indicate that: (1) ancient wise men priming effects are saliently higher than modern wise men priming effects; (2) ancestor priming and modern men priming belong to different psychological mechanisms; and (3) assimilation effect and contrast effect appear in intelligence priming effects. In conclusion, ingroup ancestor priming, i.e., ancestor advantages, indeed can motivate people's better intellectual performances.
\end{abstract}

Keywords: intelligence priming effect, Savanna principle, assimilation effect, contrast effect

\section{Introduction}

Evolutionary psychology believes that the psychological mechanisms of intelligence priming are of strong survival significance. Survival and growth are the most primary problems of adaptability in the process of human evolution, and meanwhile generate corresponding adaptive psychological mechanisms. Large amounts of evidence indicate that $99 \%$ of men's evolutionary history was during the hunter-gatherer savanna social form, so modern humans possess many psychological mechanisms, such as phobias of snakes, spiders and strangers, preference of landscape similar to savannas, and willingness of accepting and helping closer relatives, etc., implying huge amounts of information of ancestors' survival. This demonstrates that we have evolved domain-special psychological mechanisms in response to ancient survival rather than to modern society, which is defined as ancestor advantages. Further, humans also evolved a few domain-general psychological

\footnotetext{
* This research was funded by the project of Humanities and Social Sciences of the Ministry of Education of China (13YJA190006).

Zhang Shu (Correspondence author), Ph.D. candidate, School of Psychology, Shandong Normal University. Email: 1227manman@163.com

Guo Qingke, Ph.D., School of Psychology, Shandong Normal University.
} 
mechanisms, such as general intelligence, etc.. Chiappe and MacDonald argued that, domain-general mechanisms like general intelligence are evolved to "solve unusual problems in the evolutionary process" or to develop novel solutions to old problems (Chiappe \& MacDonald, 2005; Geary \& Huffman, 2002). However, the psychological mechanisms of intelligence priming are the superordinate mechanisms that humans might possess to regulate other mechanisms instead of merely domain-special or domain-general psychological mechanisms. Apparently, evolved intelligence priming mechanisms often interact in a very complicated fashion, and prime general intelligence in different backgrounds so as to enable people to demonstrate different intellectual performances under different priming conditions.

Behavioral changes caused by priming stimuli (stereotype, attribute, attitude, objective, etc.) are due to the existence of specific perception-behavior connection between priming stimuli and behavioral schemas; once behavioral perception is primed in memory, real behaviors will be automatically triggered, including complex social behaviors and intelligent behaviors (Bargh \& Huang, 2009). By priming the concept of "professor", Dijksterhuis et al. discovered that people tend to demonstrate higher accuracy in a general knowledge test than the control group, whereas priming the concept of "soccer hooligan" tends to cause worsen intellectual performances of the subjects (Dijksterhuis \& Van Knippenberg, 1998). This is because priming stimulus of "professor" primes high intelligence related schemas in the subjects' minds, enabling them to devote more willpower and efforts in answering questions, employ more sapient resolution strategies, and try additional flows of thoughts with more concentration and confidence, thereby giving rise to the phenomena of accuracy increase.

Research has discovered that the relationship between priming and intellectual behavior does not present in a stable way: in the research by Shanks et al., the subjects in priming group of "professor" failed to score higher than other control groups, while priming group of "soccer hooligan" didn't score lower than that of "professor" (Shanks et al., 2013). For this phenomenon, this study holds that (1) intelligence priming effect is subject to the characteristics of people themselves, in which different people demonstrates different personal relationship with the same priming stimulus (Bartlett, 2013); the more correlated priming stimuli to people, the more evident the priming effects (Mandel, Petrova, \& Cialdini, 2006); and (2) the investigative approaches to intelligent priming effects are not ideal, which commonly use general knowledge questions and general intelligence tests. Due to difference in cultural backgrounds, test of general knowledge questions is not suitable for subjects from all countries, while the abstractness and direct immeasurability of general intelligence itself bring about difficulties to general intelligence identification.

From the perspective of evolutionary psychology, this research proposes several improvements of previous researches. The first is to introduce ancestor savanna survival priming conditions. Intelligence is the product of evolutionary adaptation to the environment during the process of human evolution (Cosmides \& Tooby, 2002). The majority of human cognition took shape in the Pleistocene about 1800,000 to 10,000 years ago, in which period the human beings inhabited the African Savannah and lived on hunting and gathering. Psychological mechanisms are designed to adapt to the "environment of evolutionary adaption"-ancestor survival environment rather than the current environment, and so are the human brain mechanisms. This is called the "Savanna principle", that is, it is difficult for human brains to understand and deal with matters and scenes nonexistent in the ancestral environment (Kanazawa, 2004). The second proposal of this research is to improve evaluation tools. Evolutionary psychologists suggest that volatile and unstable information patterns 
generated by dynamic social interactions give rise to human abilities of dealing with uncertain scenes, inhibiting stylized responses, and coming up with novel methods to solve problems, which are referred to as general fluid intelligence in evolutionary psychology (Geary, 2005), namely, abilities displayed in the information processing and problem-solving, based on neurophysiology and not subject to education and culture. Geary argued that general fluid intelligence and the component of cognitive processing are psychological abilities of solving novel problems in face of environment of adaptive evolution (Geary, 2005). In this research, Raven Intelligence Test is used to conduct evaluation together with cognitive processing tasks. In consideration of timeliness of priming effect, the fifth set of Raven Intelligence Test is selected according to previous research on Raven's test and Raven's Standard Progressive Matrices. The test includes five series of cognitive attribute levels, and the fifth set mainly focuses on capability for abstract reasoning, closer to the general intelligence content investigated in this research.

Besides, the impacts of group bias on intelligence priming are investigated in this research. Human tendency of recognizing others as members of specific groups is a sort of effective social cognition process (Macrae \& Bodenhausen, 2000). Under certain specific conditions, humans would display preset negative attitudes toward outgroup members, but the biases are not completely negative or useless. In the long term, group bias can be regarded as an evolved psychological mechanism capable of increasing individual chances of survival (Fishbein, 2010). As a country of collectivist culture, China takes family as its basic social structure and functional unit (i.e., "the most natural ingroup"). Thus, to be more suitable for Chinese subjects, this research utilizes the degree of family correlation to divide ingroup/outgroup members.

\section{Preliminary Experiment}

Processing basics of general cognitive ability includes working memory and processing speed (Burns, Nettelbeck, \& McPherson, 2009). In the preliminary experiment, E-prime is used to program working memory, processing speed and other cognitive processing tasks to evaluate general cognitive ability.

\section{Research Method}

Subjects: 436 high school students of the first year with half male and half female.

Experiment procedures: tests of cognitive processing tasks, namely working memory test and processing speed test. E-prime is programmed, instruction presented and exercises provided; formal evaluation is conducted after all subjects passed the exercises. Raven Intelligence Test is a written test, selecting the fifth set with 12 questions.

\section{Project Analysis}

Delete question items with the total correlation under 0.4, determine 31 questions in the working memory test and 30 questions in the processing speed test with the total correlation between 0.53 and 0.88 and the $\alpha$ coefficient between 0.82 and 0.90. According to KMO (0.78) and Bartlett's Test of Sphericity (chi-square value $=1.723 \mathrm{E} 4, p<0.05)$, the data is suitable for factor analysis. A common factor of the eigenvalue above 1 is extracted and the factor loadings of the items are between 0.42 and 0.66 , which indicates that the factor model is consistent with the intelligence structure proposed above. In other words, the Raven Intelligence Test, working memory test and the processing speed test are all loaded on this common factor, which is thereby determined as the general fluid intelligence component. 


\section{Experiments}

This research adopts various intelligence priming scenes and the groups correlated and not correlated to family in conducting experiments so as to further explore what kinds of priming advantages different intelligence priming scenes would demonstrate on working memory, processing speed, spatial reasoning and other cognitive processing abilities.

\section{Experiment 1}

Experiment objectives. Objectives of Experiment 1 are to introduce ancestor savanna survival priming conditions on the basis of existing researches, compare the impacts of ancestor savanna survival priming, professor priming, soccer hooligan priming and non-priming control conditions on intelligence priming effect, and investigate the influence of ingroup/ougroup on intelligence priming from the family correlation.

Research method. Subjects: A total of 310 high school students of the first year participate as subjects in the experiments and each class is randomly allocated with a priming scene.

Experiment materials: the Raven Intelligence Test and working memory and processing speed evaluation procedures programmed by Eprime 2.0 software. For specific evaluation steps, see the preliminary experiment.

Experiment design: A completely random variance design of 4 (priming conditions: ancestor, professor, soccer hooligan and control condition) $\times 2$ (family correlation: in-group, out-group), in which the both variables of the priming conditions and degrees of family correlation are considered as between-subject variables.

Experiment procedures: (1) Priming phase: With written texts demonstrating different prototype priming (ancestor priming, professor priming, soccer hooligan priming, control group), subjects are required to enumerate typical behaviors, lifestyles and personality traits of the prototypes according to the instructions; this phase lasts for 15 minutes; (2) Examination phase: Intelligence tests are conducted after the completion of priming task, including Raven Intelligence Test, working memory test and processing speed test; this phase takes 30 minutes; and (3) Inspection phase: The subjects are required to answer two questions: (a) what do you think are the objectives of this research? (b) Is there any influence of the first written task on the later computer task? If there is, what is it? This phase is to examine whether the subjects perceive the objectives of the research and the impacts of the former priming phase on the following examinations.

Results analysis. $4 \times 2$ completely random variance analyses are conducted respectively.

As to the index of reaction time (RT), there exists salient interaction between the priming conditions for the reaction time of cognitive processing task and family correlation, $F(2,264)=4.86, p<0.01, \eta^{2}=0.35,1-\beta$ $=0.76$ : family correlation effect of ancestor priming is salient, $F(1,266)=11.33, p<0.01$, while other effects are non-salient (see Figure 1). There exists salient interaction effect between priming conditions for the reaction time of working memory and family correlation, $F(2,266)=5.06, p<0.05, \eta^{2}=0.36,1-\beta=0.77$ : family correlation effect of ancestor priming is salient, $F(1,267)=12.31, p<0.01$; while other effects are non-salient (see Figure 2).

In order to balance the relationship between accuracy and speed, the researcher proposes the concept of B value $(\mathrm{B}=$ total score/ total $\mathrm{RT}$ ). $\mathrm{B}$ value reflects the efficiency of intelligence cognitive processing, in proportion to total score and in inverse proportion to total RT. For people of the same degree of accuracy, the less the RT, the higher the B value, and the higher the level of intelligence, vice versa. Therefore, a higher B value indicates a better intelligence performance. Since the index of RT in the working speed tasks per se 
represents intelligence efficiency, this research only investigates B values in the cognitive processing tasks and working memory tasks.

As to the index of intelligence efficiency (IE), the main effect of priming conditions for cognitive processing tasks is salient, $F(2,275)=3.63, p<0.01, \eta^{2}=0.38,1-\beta=0.68$; ancestor priming is higher than professor priming $(M D=0.52, p<0.01)$ and control priming $(M D=0.51, p<0.01)$. There are salient interactive effects between the priming condition and family correlation, $F(2,275)=3.38, p<0.05, \eta^{2}=0.24$, $1-\beta=0.87$; there exists salient effect for family correlation of ancient priming, $F(1,277)=7.56, p<0.01$; and there exists salient effect for family correlation of professor priming, $F(1,277)=4.65, p<0.05$. The out-groups ancestor priming is much higher than the level of interaction between other priming scenes and family correlation (see Figure 3).

There exists marginal salience in the main effects for the working memory priming conditions, $F(3,284)=$ $2.61, p=0.051, \eta^{2}=0.027,1-\beta=0.57$; ancestor priming is higher than professor priming $(M D=0.24, p=$ $0.063)$. There is salient interaction effect between priming conditions and family correlation, $F(2,284)=3.01$, $p<0.05, \eta^{2}=0.21,1-\beta=0.73$ : salient effects exist for family correlation of ancestor priming, $F(3,284)=5.11$; $p<0.01$, salient effects exist for family correlation of professor priming, $F(3,284)=3.11, p=0.05$; while other effects are non-salient. Out-group Ancestor priming groups is saliently higher than the level of interaction between other priming scenes and family correlation (see Figure 4).

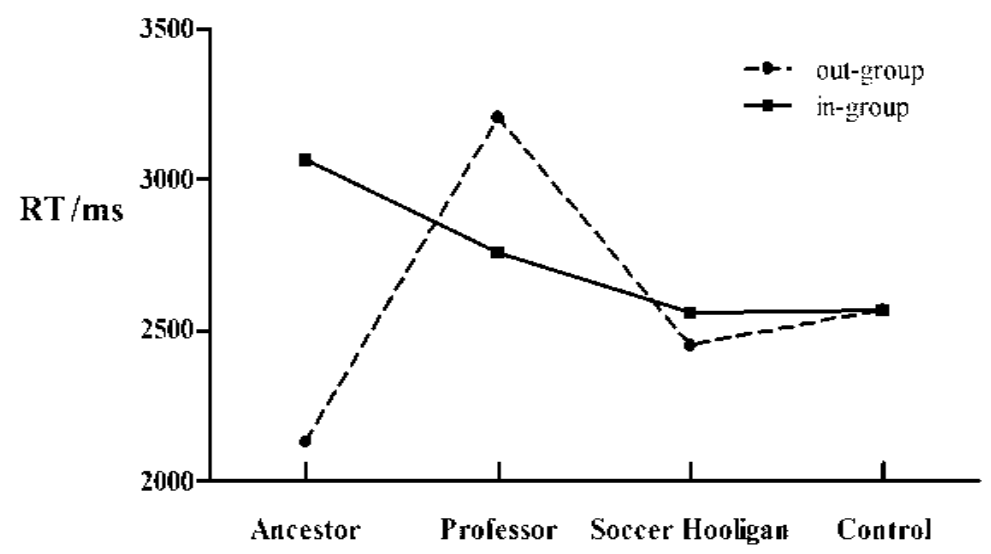

Figure 1. Reaction times in the cognitive processing task under priming conditions.

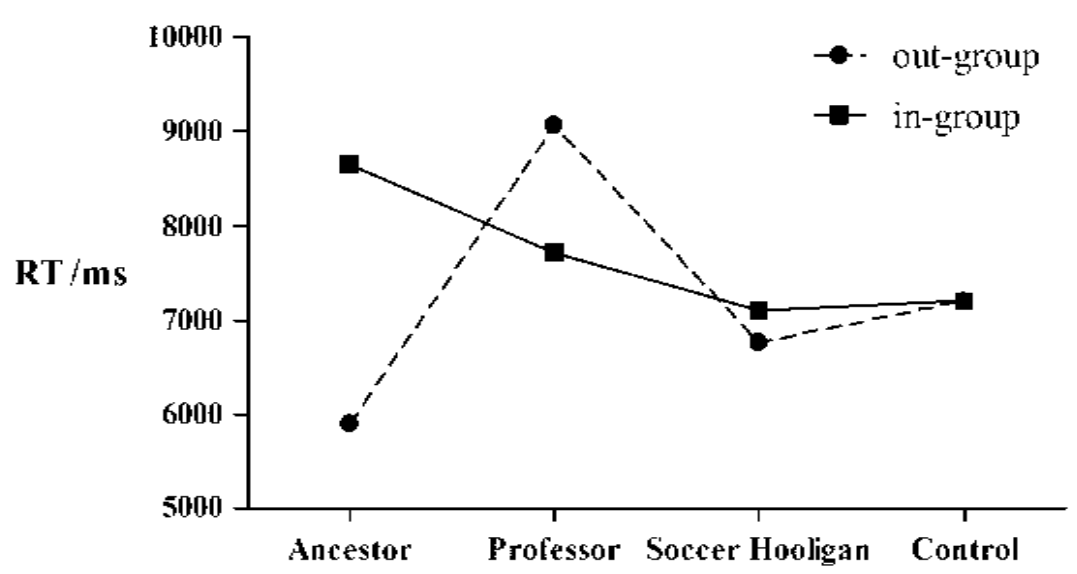

Figure 2. Reaction times in the working memory task under priming conditions. 


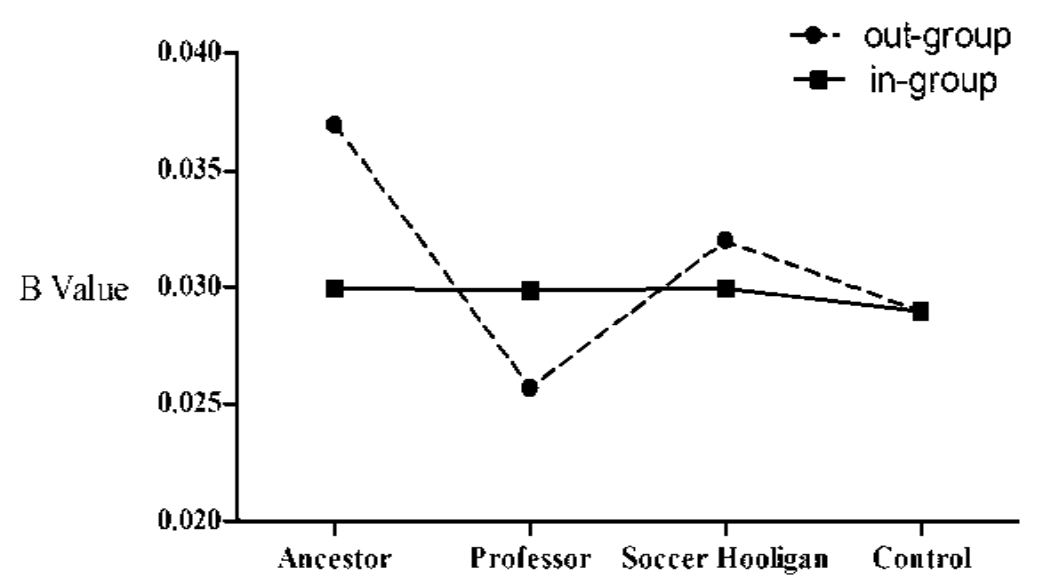

Figure 3. Values in the cognitive processing task under priming conditions.

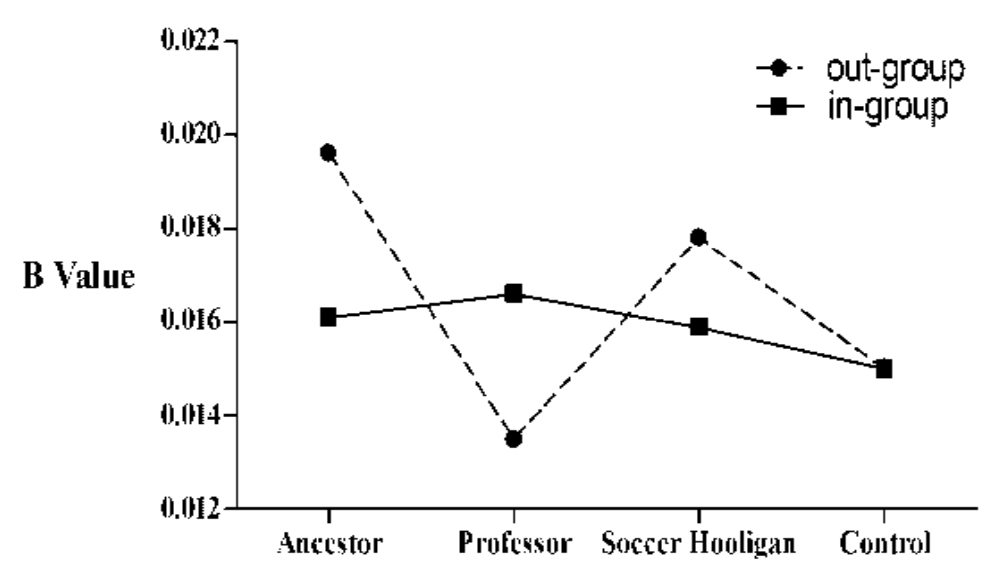

Figure 4. Values in the working memory task under priming conditions.

Discussion. Research results show that intelligence priming effects of the image of ancestor are saliently better than those of the image of modern men as anticipated. However, professor priming effects of family correlated groups (ingroups) exhibit salient difference with those of non-family control groups, inconsistent with anticipation. This indicates that in the priming of non-family control groups, the subjects tend to classify professor as an outgroup member, which could be owed to negative stereotypes of professor in the current society. In the meantime, based on existing research (Dijksterhuis et al., 1998), high intelligence priming of outgroups will decrease intelligence performances of the subjects, which could suggest that the subjects are of negative attitudes toward professor and classify professor as an outgroup member. Soccer hooligan priming demonstrates non-salient differences among different family correlation, which is consistent with the non-priming control group; this is because the Chinese subjects are not familiar with the image of soccer hooligan. In order to improve the insufficiencies of Experiment 1 to be more suitable for the subjects of Chinese high school students, Experiment 2 is designed.

\section{Experiment 2}

Experiment objectives. Objectives of Experiment 2 are to change the high intelligence priming (professor priming) and low intelligence priming (soccer hooligan priming) in Experiment 1 into top student priming and below average student priming, so that the priming images are more familiar to Chinese high school students. 
In this way, the experiment will explore the influence of the conditions of ancestor savanna survival priming, top student priming, below average student priming and non-priming control on intelligent priming effects, as well as investigate ingroup/outgroup impacts on intelligence priming from the angle of family correlation.

Research method. Subjects: 316 high school students of the first year are taken as subjects, with each class randomly allocated with a priming scene.

Experiment materials: The same as in Experiment 1.

Experiment Design: A completely random variance design of 4 (priming conditions: ancestor, top student, below average student, control) $\times 2$ (family correlation: family correlated group, non-family control group) Both priming conditions and degrees of family correlation are considered as variables among the subjects.

Experiment procedures: priming conditions include ancient ancestor priming, top student priming, below average student priming and control group. Other procedures are the same as those in Experiment 1.

Results analysis. $4 \times 2$ completely random variance analyses are conducted respectively.

As to the index of reaction time, there exist salient main effects of family correlation for the reaction time of cognitive processing task, $F(2,271)=4.18, p<0.01, \eta^{2}=0.15,1-\beta=0.53$. Non-family control groups are lower than family correlated groups. There appear salient interaction between the priming conditions and family correlation, $F(2,271)=3.72, p<0.05, \eta^{2}=0.27,1-\beta=0.68$ : the effect for family correlation of ancestor priming is salient, $F(1,273)=12.03, p<0.05$; the effect for family correlation of below average student priming is also salient; while other effects are non-salient (see Figure 5).

As to the index of accuracy, the accuracy results of the Raven Intelligence Test suggest that none of the effects are salient, which is consistent with Shanks et al.'s results (2013), but their trends are consistent with those of total scores of cognitive processing, total scores of working memory and results of Experiment 1. There exist salient interactive differences in the total scores of the cognitive working task, $F(2,281)=4.74, p<$ $0.05, \eta^{2}=0.33,1-\beta=0.72$ : effects for family correlation of below average student are salient, $F(1,284)=5.77$, $p<0.05$; while others are non-salient (see Figure 6). There exist salient main effects for the total scores the priming conditions of working memory task, $F(3,293)=4.15, p<0.01, \eta^{2}=0.041,1-\beta=0.85$ : ancestor priming is higher than top student priming, below average student priming and control priming. Salient interaction is demonstrated between priming conditions and family correlation, $F(2,293)=3.75, p<0.05, \eta^{2}=$ $0.025,1-\beta=0.68$ : family correlation of ancestor priming exhibits marginal salience, $F(1,295)=3.35, p=0.06$; while others are non-salient (see Figure 7). There exist salient main effects for the total scores priming conditions of processing speed, $F(3,277)=5.99, p<0.01, \eta^{2}=0.069,1-\beta=0.81$ : ancestor priming is higher than top student priming, below average student priming and control priming. These results are consistent with those of Experiment 1.

There exist salient effects for the family correlation of working memory reaction time, $F(1,282)=5.13, p$ $<0.05, \eta^{2}=0.18,1-\beta=0.62$, non family control groups are saliently lower than family correlated groups $(M D=$ $1170.03, p<0.05)$; priming conditions and family correlation demonstrate salient interaction, $F(2,282)=3.40$, $p<0.01, \eta^{2}=0.24,1-\beta=0.64$ : ancestor priming demonstrates salient family correlation effects, $F(1,284)=$ $12.29, p<0.05$; below average student priming also demonstrates salient family correlation effects, $F(1,284)=$ $4.67, p<0.05$; while others are non-salient (see Figure 8). 


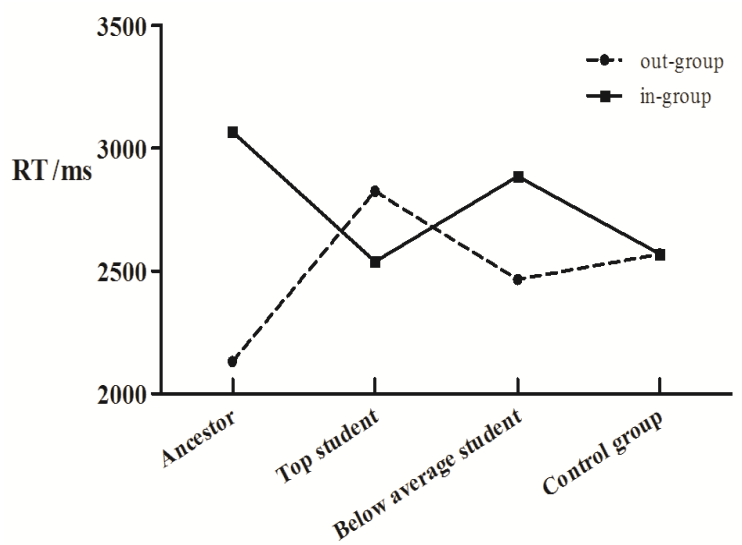

Figure 5. Reaction Times in the Cognitive

Processing Task under Priming Conditions.

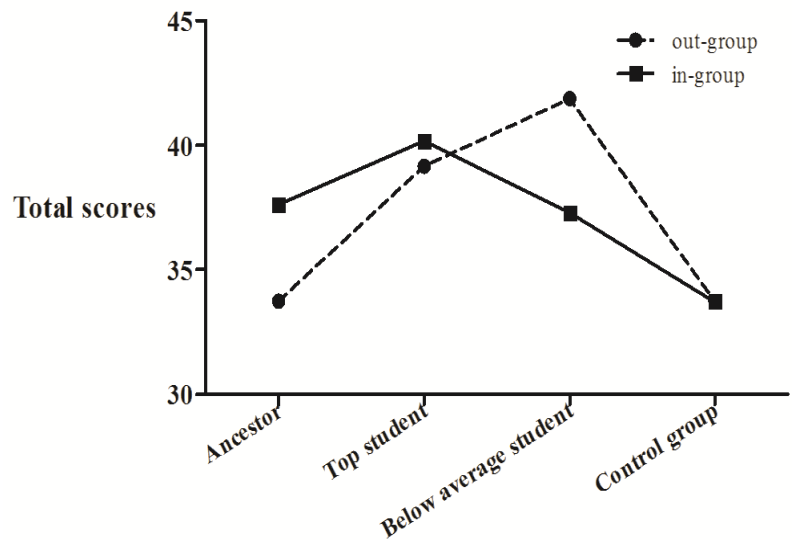

Figure 7. Total scores in the working memory task under priming conditions.

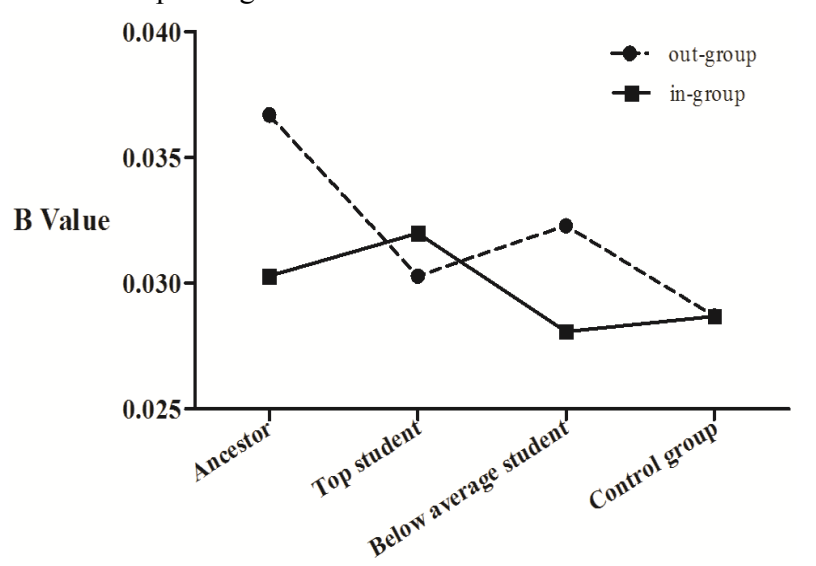

Figure 9. B-values in the cognitive processing task under priming conditions.

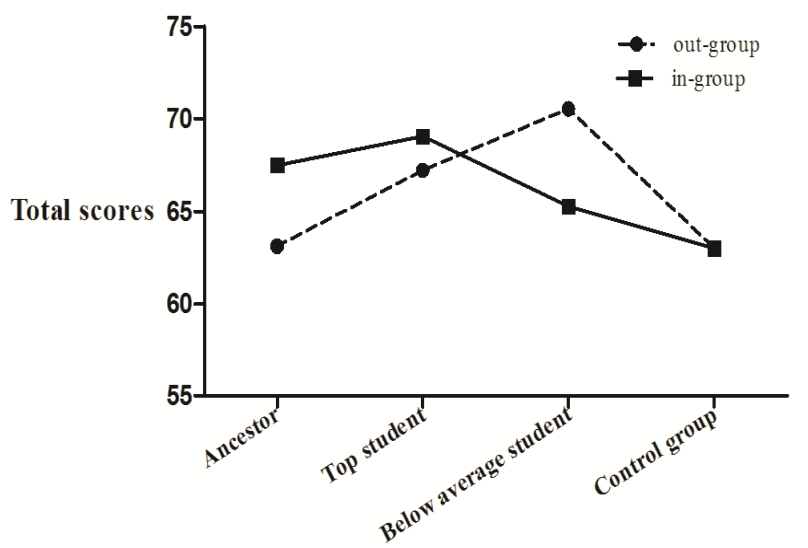

Figure 6. Total scores in the Cognitive Processing Task under Priming Conditions.

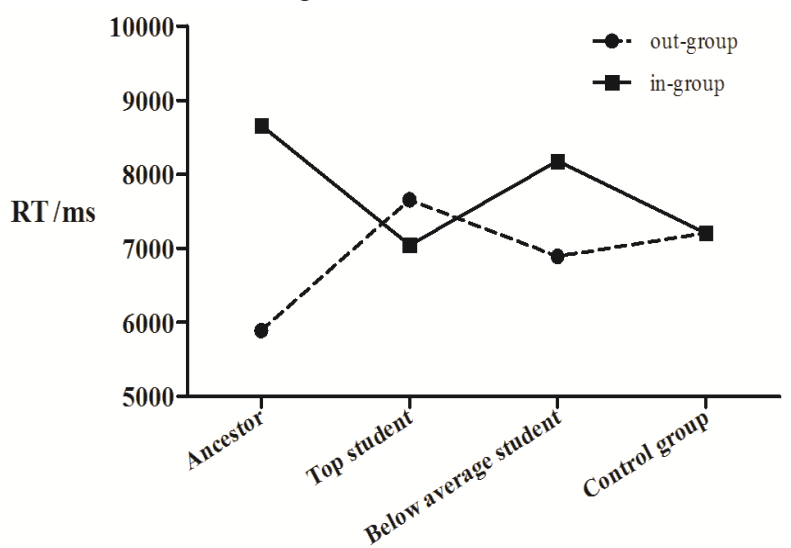

Figure 8. Reaction times in the working memory task under priming conditions.

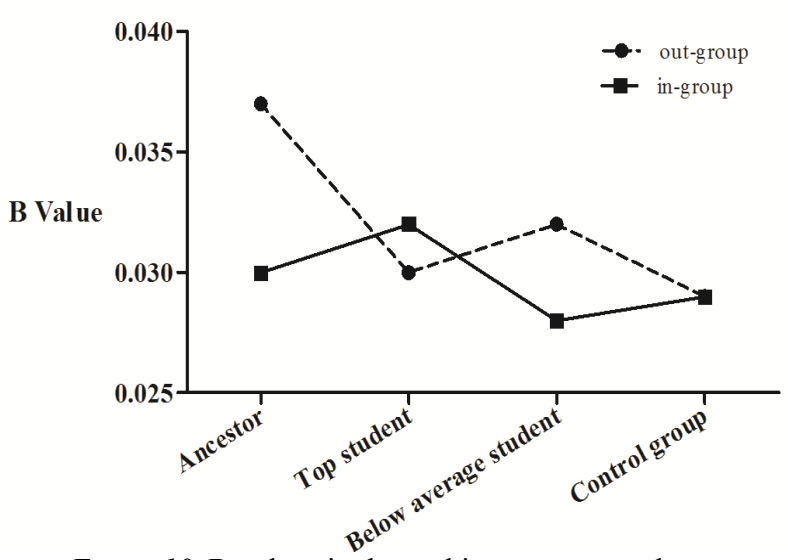

Figure 10. B-values in theworking memory task under priming conditions.

As to the index of intelligence efficiency, there are salient main effects for the priming conditions of cognitive processing tasks, $F(3,279)=2.98, p<0.05, \eta^{2}=0.31,1-\beta=0.70$; ancestor priming is saliently higher than below average student priming $(M D=0.33, p<0.05)$ and control priming $(M D=0.51, p<0.05)$. Salient interactions appear between family correlation and priming conditions, $F(2,279)=2.21, p<0.01, \eta^{2}=0.24$, $1-\beta=0.63$ : the family correlation effects of ancestor priming are exhibited saliently, $F(1,281)=7.95, p<0.01$; 
family correlation of below average student priming exhibits salient marginal effects, $F(1,281)=2.72, p=$ 0.064; while others are non-salient. Meanwhile, the interactive level for ancestor priming of non-family control groups is much higher than all others. These results are consistent with those of Experiment 1 (see Figure 9).

There exist salient marginal effects for the family correlation of working memory, $F(1,292)=4.86, p=$ $0.06, \eta^{2}=0.023,1-\beta=0.74$, non family control groups are saliently higher than family correlation groups (MD $=0.2, p<0.01$ ). Under the scene of ancestor priming, non-family control groups are saliently higher than family correlation groups, $(M D=0.37, p<0.05)$; under the scene of below average student priming, non-family control groups are saliently higher than family correlated groups $(M D=0.28, p<0.05)$. Top student priming effects are non-salient (see Figure 10).

Discussion. The results also show that the intelligence priming effects of the image of ancestor are saliently better than those of the image of modern men as anticipated and as shown in Experiment 1 . Top student priming effects in family correlation groups and those in non-family control groups present non-salient differences, which suggests that the subjects demonstrate positive attitudes toward top student and tend to include it as an ingroup member. Since top student priming of family correlated groups is in effect ingorup member priming, and the priming results of non-family control groups are consistent with those of family correlated groups, it is indicated that in the priming where whether the control is a family member or not, the subjects tend to automatically classify top student as a family member, i.e., an ingorup member. For ingroup (family correlated group) priming, top student priming effects are saliently higher than below average student priming, consistent with the results of existing research that ingroup high intelligence priming is conducive to increasing the subjects' intelligence performance (Dijksterhuis, Aarts, Bargh, \& Van Knippenberg, 2000). The priming of below average students is in other way around, indicating that the subjects are of negative attitudes toward below average student and tend to classify it as an outgroup member; low intelligence priming of outgroup members is conducive to increasing the subjects' intelligence performance, which is consistent with existing research.

\section{General Discussion}

The research discovers that: (1) For accuracy, there is no salient difference of intelligence priming in the Raven Intelligence Test, which is consistent with research results by Shanks et al.. However, this research finds out that the trajectory direction of the Raven Intelligence Test scores is consistent with those of the total scores of cognitive processing and the total scores of working memory, and the results of Experiment 1 and 2 are consistent with each other; (2) For reaction time, intelligence priming effects are salient. Ancestor priming exhibits the largest differences among groups of different degrees of family correlation. Under the conditions of non-salient accuracy difference, the less the reaction time, the better the intelligence performance would be. As a result, ancestor priming of non-family control groups has the best effects. The results of Experiment 2 are consistent with those of Experiment 1; and (3) For intelligence efficiency, ancestor priming of non-family control groups exhibits the highest $\mathrm{B}$ values for cognitive processing and working memory, which are saliently higher than combinations of other priming conditions and family correlation levels. The results of Experiment 2 are consistent with those of Experiment 1.

Although the intelligence priming effect remains a topic of constant controversy, from the results of this research, it is seen that there indeed exists intelligence priming effects, and intelligent priming of the image of ancestor exhibits better effects than that of the image of modern men. 
Form the results of this research, it can be seen that intelligent priming effects of the image of ancestor are better than those of the image of modern men, while different family correlated groups display different priming effects, the reason of which lies in that the two types of intelligence priming belong to different psychological mechanisms.

Under the scene of modern men priming, people will classify the priming prototype into either ingroups (family correlated groups) or outgroups (non-family correlated groups) according to their own degrees of recognition of it. When people identify the example as an ingroup member, their self schemas will activate the assimilation effect, that is, priming prototype of positive traits will lead to higher intelligence performance, and the priming prototype of negative traits will lead to lower intelligence performance. When people identify the example as an outsider or outgroup member, contrast effects will emerge, that is, prior priming will cause reverse influence on later judgments. For instance, for the index of intelligence efficiency, the professor priming in Experiment 1 and the below average student priming in Experiment 2 demonstrate salient differences between the two family correlation levels. As subjects of non-family control groups have lower degree of recognition toward below average student and tend to classify below average student into non-family correlated groups, there exist salient differences between the two family correlation levels. The same is in the case of professor priming. On the contrary, subjects of non-family control groups have higher degree of recognition toward top student and tend to classify top student into family correlated groups, so there exists no difference between the two family correlation levels of top student priming, which is consistent with prior research (Gordijn \& Stapel, 2006). Since soccer hooligan priming has the priming prototype unrelated to the self-schemas of Chinese high school students, there is no effect exhibited by this priming.

The case is, however, quite different for ancestor priming. At the level of non-family control groups, the subjects tend to classify ancestor into non-family correlated groups, i.e., outgroups. According to signal detection theory, humans' cognitive biases under uncertain scenes are favorable for their survival and reproduction. Whether individuals will make false or omitted cognitive biases hinges on their cost. The kind of bias of less cost and larger chances of survival will be retained by natural selection. In this study, under priming condition of non-family control groups, individuals are more likely to classify ancestor into non-family correlated groups, that is, to make the mistake of false bias, the reason of which lies in that in the case of omitted bias of judging enemies as family, the individual might lose his or her life. Further, ancestor priming in non-family correlated groups gives rise to significantly lessened reaction time but non-significant accuracy decrease in people and their intelligence efficiency is saliently higher than all other combinations of levels. The researcher holds that this is because of the presence of the "intergroup antagonism" mechanism with family as the basic group in human brains, which is primed by the image of ancestor. From the evolutionary angle, outgroup members pose a potential threat, which, when faced by individuals in an antagonistic state, will prime the intergroup antagonism as a protection mechanism (Brewer, 1999) in defense of the individuals from attacks by outgroup members (Cosmides, Tooby, \& Kurzban, 2003). At that time, people tend to overestimate potential threats, and make response and judgment within the minimum time, discarding part of the accuracy so as to increase intelligence efficiency. The cost of the formation and maintenance of such harm-aversion psychological mechanism is far less than actual harm, which is favorable for the survival of the group. This is, namely, the evolutionary preparatory learning effect. People are more prone to responding to the stimuli related to ancestor living environments (Olsson, Ebert, Banaji, \& Phelps, 2005) and this kind of psychological mechanism is primed by ancestor intelligence priming. 


\section{Conclusions}

The intelligence priming effects of ancestors in non-family groups are saliently higher than all other combinations of priming conditions and levels of family correlation, which suggests the presence of intelligence priming effect and the Savanna principle. As for accuracy, although in both experiments the Raven Intelligence Test results, scores of cognitive processing tasks and scores of working memory show non-salient differences, they demonstrate the same direction of data trajectory, verifying the existence of intelligence priming effect in some way, which, however, shows weakly positive dimension values in terms of accuracy. The B value analytical approach balances the contradiction between accuracy and speed in the intelligence evaluation, reflecting intelligence efficiency with more effectiveness and sensitivity. In both experiments, the test scores of family groups and non-family groups, i.e., accuracy, exhibit differences in all the priming conditions. However, intelligence efficiency of family groups demonstrates non-salient differences among the priming conditions, while intelligence efficiency of non-family group displays significantly enlarging differences among the priming conditions, revealing that degree of family correlation as an important factor has influence on intelligence performance.

\section{Questions and Prospects}

This research adopts the written Raven Intelligence test and computer-utilized cognitive processing task program in investigating intelligence priming effects. Because the Raven Intelligence test in paper-pencil form only records information about accuracy without information about reaction time, part of the data is absent in the result analysis of the research. In following researches, the Raven Intelligence test should also be written into the program so as to simultaneously evaluate the accuracy and speed of Raven Intelligence Research on functionality of intelligence. Over a long period, the vast majority of intelligence-related researches have utilized structuralism analysis methods in studying the structure and operation mechanism of intelligence, but few have considered the ultimate functional values of intelligence. Research concerning intelligence should emphasize its functional values so as to better understand its structure and operation mechanism. Humans' intellectual structure is designed to take advantage of the past to serve the present or predict the possibilities of future happenings. Currently, researches on adaptive performances of intelligence in these aspects are less seen, so there still has a vast field waiting for further exploration. Currently, as the existing intelligence priming related images are all presented in the form of male, in the following researches, the impacts of gender factor on intelligence priming could be open for investigation.

\section{References}

Bargh, J. A., \& Huang, J. Y. (2009). The selfish goal. In G. B. Moskowitz, \& H. Grant (Ed.), The psychology of goals (pp. 127-150). New York, N.Y.: The Guilford Press.

Bartlett, T. (2013). Power of suggestion: The amazing influence of unconscious cues is among the most fascinating discoveries of our time-That is, if it's true. The Chronicle of Higher Education. DOI:10.1080/00987913.1977.10763009

Brewer, M. B. (1999). The psychology of prejudice: Ingroup love and outgroup hate? Journal of Social Issues, 55(3), 429-444.

Burns, N. R., Nettelbeck, T., \& McPherson, J. (2009). Attention and intelligence. Journal of Individual Differences, 30(1), 44-57.

Chiappe, D., \& MacDonald, K. (2005). The evolution of domain-general mechanisms in intelligence and learning. The Journal of General Psychology, 132(1), 5-40.

Cosmides, L., \& Tooby, J. (2002). Unraveling the enigma of human intelligence: Evolutionary psychology and the multimodular mind. The Evolution of Intelligence (pp. 127-150). New York, NY: Cambridge University Press.

Cosmides, L., Tooby, J., \& Kurzban, R. (2003). Perceptions of race. Trends in Cognitive Sciences, 7(4), 173-179. 
Dijksterhuis, Ap., Aarts, H., Bargh, J. A., \& Van Knippenberg, Ad. (2000). On the relation between associative strength and automatic behavior. Journal of Experimental Social Psychology, 36(5), 531-544.

Dijksterhuis, Ap., Spears, R., Postmes, T., Stapel, D., Koomen, W., Van Knippenberg, Ad., \& Scheepers, D. (1998). Seeing one thing and doing another: Contrast effects in automatic behavior. Journal of Personality and Social Psychology, 75(4), 862.

Dijksterhuis, Ap., \& Van Knippenberg, Ad. (1998). The relation between perception and behavior, or how to win a game of trivial pursuit. Journal of Personality and Social Psychology, 74(4), 865.

Fishbein, H. D. (2010). The genetic/evolutonary basis of prejudice and hatred. Journal of Hate Studies, 3(1), 113.

Geary, D. C. (2005). The origin of mind. American Psychological Assoc.

Geary, D. C., \& Huffman, K. J. (2002). Brain and cognitive evolution: Forms of modularity and functions of mind. Psychological Bulletin, 128(5), 667.

Gordijn, E. H., \& Stapel, D. A. (2006). Retracted: Behavioural effects of automatic interpersonal versus intergroup social comparison. British Journal of Social Psychology, 45(4), 717-729.

Kanazawa, S. (2004). The savanna principle. Managerial and Decision Economics, 25(1), 41-54.

Macrae, C. N., \& Bodenhausen, G. V. (2000). Social cognition: Thinking categorically about others. Annual Review of Psychology, 51(1), 93-120.

Mandel, N., Petrova, P. K., \& Cialdini, R. B. (2006). Images of success and the preference for luxury brands. Journal of Consumer Psychology, 16(1), 57-69.

Olsson, A., Ebert, J. P., Banaji, M. R., \& Phelps, E. A. (2005). The role of social groups in the persistence of learned fear. Science, 309(5735), 785-787.

Shanks, D. R., Newell, B. R., Lee, E. H., Balakrishnan, D., Ekelund, L., Cenac, Z., ...Moore, C. (2013). Priming intelligent behavior: An elusive phenomenon. PloS one, 8(4), e56515. 